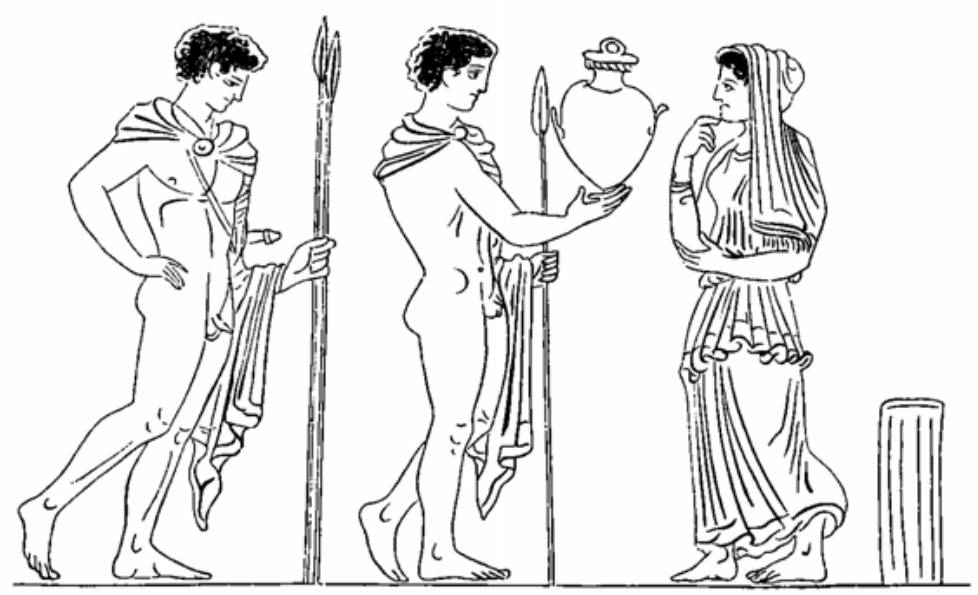

\title{
PRAEFATIO EDITIONIS SECVNDAE (MDCCCLXXII)
}

Inter extrema mandata quae Oтто IAHNIvs iam in ipso mortis limine versans mihi dedit hoc quoque erat, ut Electrae sophocleae abhinc annos undecim ab ipso editae novam curarem editionem non mutato universo libri consilio sed multo locupletiore apparatu instruendam. cum enim magis magisque dubitare coepisset, essetne laurentianus L communis omnium quotquot hodie extant codicum sophocleorum fọns, Hvgonem Hinckivm precibus adierat ut laurentianos 1 et $\mathrm{G}$ in suos usus conferret; quo munere ille paratissimo animo suscepto ita functus est, ut praeterea ipsum quoque $\mathrm{L}$ consuleret ubicumque vel aliqua de causa de Duebneri fide dubitaret vel mutatam a diversis correctoribus pristinam libri scripturam videret, ut tandem de partibus correctorum $\mathrm{L}$ L $\mathrm{L}$ L quam certissime fieri posset constaret. pergrate deinde acciderat ut Rvdolfvs Prinzivs quam sibi confecerat codicum parisinorum $\mathrm{P}$ et $\mathrm{E}$ collationem eam praeceptori utendam ultro offerret; neque post Iahnii mortem idem mihi defuit ut in illius iura succederem petenti. denique EDvardvs HiLlervs a Iahnio rogatus facile pietati dederat ut librum vindobonensem identirlem ab Heimsoethio laudatum excuteret. quibus copiis monente Iahnio ita usus sum, ut librorum 
florentinorum parisinorumque plenam adscriberem varietatem (nisi quod neque iota mutum in PEG plerumque omissum neque

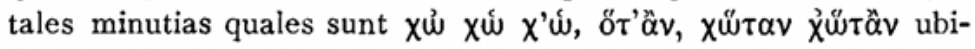
que curavi et lyricorum dispositionem e solo $L$ enotavi); ex vindobonensi libro, qui praeter pauca ingeniose emendata interpolationibus mendisque e librarii neglegentia ortis scatet, quae vel ad emendandum Sophoclem valere vel ad recte iudicandum de codicis illius indole sufficere videbantur excerpsi. neque vero fefellisse Iahnium opinionem quam de codicum ratione conceperat non dubito quin concessuri sint qui adnotationem criticam accuratius examinaverint. patet enim recte a quibusdam viris doctis duplex librorum sophocleorum genus statui, ab altera parte stare praestantissimos L l, de quibus quae Hinckivs observavit infra leges, ab altera parte $\mathrm{PE} \mathrm{L}^{2}$, medium quendam inter utrosque locum tenere florentinum G; qua de re Prinzivs uberius expositurus est. - ceterum in constituendis poetae verbis editionis scholarum maxime usui destinatae rationem ita rectissime servari ratus sum, ut ab optimorum librorum auctoritate ne in aperta quidem corruptela recederem, nisi si emendatio suppeteret vel certa vel omnes probabilitatis numeros habens; nec dissimile consilium in lyricis secutus sum laurentiani codicis dispositione quoad ratio permittere videbatur servata. ne quem tamen quid Iahnius probaverit lateat indice extremae tragoediae subiecto cautum est. codicum scripturis quod virorum doctorum coniecturas et olim ab editore collectas et novas quotquot mihi innotuerunt addidi, id non tam meum arbitrium secutum quam a Iahnio iussum me fecisse scito, quippe qui lectorum commodis melius consultum iri existimaret si congesto toto acervo pro suo cuique iudicio facilis optio daretur, quam si improbus ista conquirendi labor unicuique denuo suscipiendus relinqueretur. praeter ipsa auctorum nomina plerumque librorum libellorumve locos accurate indicatos invenies. denique tam scholia quam vitam poetae recognovi, in hac operis parte novis subsidiis adiutus ab Hillero et Prinzio et Rvdolfo Schoellio paratis; eodem Schozllio officiose intercedente Florentiae specimen libri laurentiani versus $357-404$ complectens solis ope exceptum est, HILLERI autem prompta comitas in corrigendis typothetae erroribus mihi adfuit.

restat ut subiciatur 
Hvgonis Hincki de libris L et l dispvtativncvla.

'Laurentianum 1, quamquam omnium quos novimus codicum ad L proxime accedat, non tamen ex ipso hoc libro descriptum esse et discrepantiarum magnus numerus ratioque et personarum laterculus non omissus ostendunt. quod si animadverteris l tum cum L L L $\mathrm{L}^{\mathrm{x}}$ tum cum $\mathrm{L}^{2}$ consentire, forsitan speres illius ope ad distinguendas varias manus, quas uno signo $\mathrm{L}^{2}$ complectimur, te esse perventurum; quas manus ut per quasdam codicis partes facile persequeris, ita singulis quibusque locis atramenti vel scripturae ope quid cuique librario debeatur certo discernere vereor ne nemini contingat. iam vero hoc constat, I nequaquam solere unam aliquam illarum manuum constanter sequi spernere alteram, sed mutationum ab eodem correctore factarum alias ab 1 adgnosci alias ignorari. unde coniciamus necesse est eum librum $(\lambda)$ e quo 1 originem traxit ex $L$ descriptum esse quo tempore hic correcturarum illarum ( $\left.\mathrm{L}^{2}\right)$ aut nullas aut paucissimas expertus sit; tum in alios libros, siqui inter $\lambda$ et $l$ intercesserunt, aut casu aut consilio inlatas esse lectiones pariter a $\mathbf{L} \mathbf{L} \mathbf{L}$ atque a $\mathrm{I}^{\mathrm{a}}$ alienas, alias e codicibus vulgaribus receptas, additum denique personarum laterculum. cum tamen plurimis locis 1 pristinam libri L scripturam servatam exhibeat, utilissimus profecto est cuius ope haec, quamquarn postea a $\mathrm{L}^{2}$ mutata et oblitterata, cognoscatur. unum superest quod moneam, nimirum omnibus eis locis ubi, quod mireris, L l contra I stant (364. 439. 449. 512. 592 ? 783. 1180. 1442. 1449) non ipsa verba ab initio scripta ab I mutata esse, sed superscriptam illis esse emendationem, versu autem 991 lineolam qua $\tau$ ŵı vocabulum ab I deletum est facile potuisse ab l neglegi. ceterum aliis locis 1 scripturam a $\mathrm{L}$ superscriptam sequitur (10. 761. 1148. 1158. 1174. 1184), semel utramque adgnoscit lectionem (414). quidquid denique $\mathrm{L}$ ipsi $\mathrm{L}$ paene aequalis correxit consentaneum est ita in 1 quoque inveniri.'

His quae abhinc decem annos secundae editioni praemisi nunc, cum dudum divenditis illius editionis exemplis novam editionem emitto, pauca addenda esse video. neque enim duplex illud libelli consilium, ut et ad scholarum usum aptus esset 
et omnes virorum doctorum coniecturas sub uno conspectu poneret, iam eadem ratione atque olim factum est adsequi licuit,

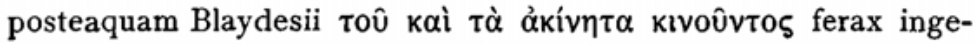
nium hanc quoque tragoediam inexhaustis inventorum suorum copiis obruit. quas si omnes commentario critico inseruissem, scholis academicis bic liber inservire non amplius potuit. itaque ut hoc quod maxime intererat servaretur, non nimis severo delectu habito eas virorum doctorum sive emendationes sive coniecturas quae aut aliquam veri speciem prae se ferre aut ad iudicia adulescentium acuenda facere viderentur una cum varietate codicum ipsis poetae verbis subicere placuit, reliquas vero in appendicem relegandas esse censui. cuius quidem supplementi ambitum intuentem iam me paenitere fateor, quod non saltem meros ingenii lusus omnique arte carentia conamina omiserim; item, si denuo res instituenda esset, commentarium criticum plurimis istis erroribus futtilibus exonerarem quibus maxime librorum $\mathrm{E}$ et $\mathrm{G}$ scribae verba poetae inquinarunt. interim commodis lectorum consuluisse mihi videor diverso scripturae genere adhibito, quo et codicum lectiones a coniecturis recentiorum et scholia antiqua a ceteris veterum grammaticorum interpretamentis distinguerentur. specimen illud photolithographum libri laurentiani quod secundam editionem ornabat fracto vitro huic editioni addere non licuit, quod non tantopere dolendum esse videtur cum et una Sophoclis pagina accurate expressa extet apud Wattenbachium et Velsenum exempl. cod. graec. tab. XXXIV et Angli cum maxime totum librum solis ope exceptum edere moliantur. contra ut imaginum aliae perfectiore cultu nunc niteant aliae novae accesserint bibliopolae honestissimi liberalitati debetur. denique publice quoque mihi gratiae agendae sunt et Henrico Grlzero, qui a me rogatus vitam Sophoclis denuo cum codice ienensi contulit, et Rvdolfo Schokllio, nunc collegae coniunctissimo, qui in corrigendis plagulis opem suam mihi deesse noluit.

Scribebam Argentorati d. xx m. Octobris a. MDccclxxxII.

Ad. Michaelis. 